\title{
Hamilton-Jacobi Method for Mechanical Systems on Time Scales
}

\author{
Xiang-Hua Zhai ${ }^{1}$ and Yi Zhang $\mathbb{D}^{2}$ \\ ${ }^{1}$ School of Science, Nanjing University of Science and Technology, Nanjing 210094, China \\ ${ }^{2}$ College of Civil Engineering, Suzhou University of Science and Technology, Suzhou 215011, China
}

Correspondence should be addressed to Yi Zhang; weidiezh@gmail.com

Received 26 March 2018; Revised 8 July 2018; Accepted 18 July 2018; Published 23 August 2018

Academic Editor: Sigurdur F. Hafstein

Copyright (c) 2018 Xiang-Hua Zhai and Yi Zhang. This is an open access article distributed under the Creative Commons Attribution License, which permits unrestricted use, distribution, and reproduction in any medium, provided the original work is properly cited.

\begin{abstract}
This paper presents the Hamilton-Jacobi method for integrating the equations of motion of mechanical systems on time scales. We give the criterion and four basic forms of canonical transformation on time scales. Also, various examples are given to illustrate the role played by a generating function in the canonical transformation. By choosing an appropriate generating function, we construct the Hamilton-Jacobi equation on time scales and prove the Jacobi theorem on time scales. An example for an Emden-Fowler type equation is discussed to show the application of the method.
\end{abstract}

\section{Introduction}

As it is well known, the Hamilton-Jacobi equation [1-4], that is an important nonlinear partial differential equation, represents a reformulation of classical mechanics. In addition, the Hamilton-Jacobi method is very useful in integrating differential equations of motion for the holonomic mechanical systems [5-9], the nonholonomic mechanical systems $[10,11]$, and the nonconservative mechanical systems [12]. The differential equations that can be realized by Hamilton formularization [13-15] can also be solved by the Hamilton-Jacobi method [13, 14]. Moreover, the discrete analogues of the Hamilton-Jacobi equation have been studied by Elnatanov and Schiff [16] and Lall and West [17]. Ohsawa et al. [18] developed the discrete Hamilton-Jacobi theory within the framework of discrete Hamiltonian mechanics. Until now, the Hamilton-Jacobi theory is well understood both from the continuous and discrete points of view, and it has many important applications in optimal control problems, particle physics, fluid mechanics, quantum mechanics, and cosmology. In this paper, the Hamilton-Jacobi method for mechanical systems is studied in version of time scales.

A time scale is an arbitrary nonempty closed subset of the real numbers. The calculus of time scales, which has recently attracted a lot of attention, was introduced by Hilger [19] in 1988 to unify continuous and discrete analysis. In terms of a unique formalism, this theory can deal with not only continuous and discrete analysis but also complex processes such as the control systems, impulsive dynamical systems, electromechanical systems, neural network, and economical systems [20-24]. The calculus of variations on time scales that was one of the popular topics has been well studied [25-35]. Inspired by these works, the Euler-Lagrange equations, the Hamilton canonical equations [36-38], and the Birkhoff's equations [39] were established for mechanical systems on time scales. Besides, the Noether's theorems [37-42] were established in finding conserved quantities for mechanical systems on time scales. However, it is worth mentioning that the famous Hamilton-Jacobi method also represents an important integration method. It is worth and necessary to study the Hamilton-Jacobi method on time scales.

The outline of this paper is as follows: we first present the basic definitions and properties about the calculus of time scales in Section 2. In Section 3, we give the Hamilton canonical equations on time scales. Section 4 focuses on the canonical transformation theory on time scales. In Section 5, we construct the Hamilton-Jacobi equations on time scales and prove the Jacobi theorem on time scales. Section 6 gives the conclusions and future directions of research. 


\section{Preliminaries on Time Scales}

We begin by introducing the definitions and properties on time scales needed in the sequel. More discussions and proofs can be found in $[21,24]$.

A time scale $\mathbb{T}$ is an arbitrary nonempty closed subset of the real numbers $\mathbb{R}$. For all $t \in \mathbb{T}$, the forward jump operator $\sigma: \mathbb{T} \rightarrow \mathbb{T}$ is defined by $\sigma(t)=\inf \{s \in \mathbb{T}: s>t\}$, while the backward jump operator $\rho: \mathbb{T} \rightarrow \mathbb{T}$ is defined by $\rho(t)=$ sup $\{s \in \mathbb{T}: s<t\}$, with inf $\phi=\sup \mathbb{T}$ and sup $\phi=\inf \mathbb{T}$.

A point $t \in \mathbb{T}$ is called right-dense, right-scattered, leftdense, and left-scattered if $\sigma(t)=t, \sigma(t)>t, \rho(t)=t$ and $\rho$ $(t)<t$, respectively. The graininess function $\mu: \mathbb{T} \rightarrow[0, \infty)$ is defined by $\mu(t)=\sigma(t)-t$. If $\mathbb{T}$ has a left-scattered maximum $M$, then we define $\mathbb{T}^{k}=\mathbb{T}-\{M\}$, otherwise $\mathbb{T}^{k}=\mathbb{T}$.

Let $f: \mathbb{T} \rightarrow \mathbb{R}$ be a function. Then the delta derivative $f^{\Delta}(t)$ of $f$ at a point $t \in \mathbb{T}^{k}$ is defined to be the number with the poverty that given any $\varepsilon>0$, there exists a neighborhood $U$ of $t$ (i.e., $U=(t-\delta, t+\delta) \cap \mathbb{T}$ ) for some $\delta>0$ such that

$\left|f(\sigma(t))-f(s)-f^{\Delta}(t)(\sigma(t)-s)\right| \leq \varepsilon|\sigma(t)-s|$, for all $s \in U$.

A function $f: \mathbb{T} \rightarrow \mathbb{R}$ is called rd-continuous if it is continuous at right-dense points in $\mathbb{T}$ and its left-sided limits exist (finite) at left-dense points in $\mathbb{T}$. The set of rd-continuous functions can be denoted by $\mathrm{C}_{\mathrm{rd}}$. The set of differentiable functions with rd-continuous derivative is denoted by $\mathrm{C}_{\mathrm{rd}}^{1}$.

A function $F: \mathbb{T} \rightarrow \mathbb{R}$ is called an antiderivative of $f:$ $\mathbb{T} \rightarrow \mathbb{R}$ provided $F^{\Delta}(t)=f(t)$ holds for all $t \in \mathbb{T}^{k}$. Then the indefinite integral of $f$ is defined by $\int f(\tau) \Delta \tau=F(t)+C$, where $C$ is an arbitrary constant. The definite integral is defined by $\int_{a}^{b} f(t) \Delta t=F(b)-F(a)$, for all $a, b \in \mathbb{T}$.

For delta differentiable $f$ and $g$, the next formulae hold

$$
\begin{aligned}
f^{\sigma}(t) & =f(t)+\mu(t) f^{\Delta}(t), \\
(f g)^{\Delta}(t) & =f^{\Delta}(t) g(t)+f^{\sigma}(t) g^{\Delta}(t) \\
& =g^{\Delta}(t) f(t)+g^{\sigma}(t) f^{\Delta}(t), \\
\int_{a}^{b} f(t) g^{\Delta}(t) \Delta t & =(f g)(b)-(f g)(a) \\
& -\int_{a}^{b} f^{\Delta}(t) g^{\sigma}(t) \Delta t, \\
\int_{a}^{b} f(v(t)) v^{\Delta}(t) \Delta t & =\int_{v(a)}^{v(b)} f(\bar{t}) \bar{\Delta} \bar{t}
\end{aligned}
$$

where we abbreviate $f \circ \sigma$ by $f^{\sigma}$, that is, $f^{\sigma}(t)=f(\sigma(t))$.

Let $f: \Lambda^{n}=\mathbb{T}_{1} \times \mathbb{T}_{2} \times \cdots \times \mathbb{T}_{n}=\left\{t=\left(t_{1}, t_{2}, \ldots, t_{n}\right): t_{i} \in\right.$ $\left.\mathbb{T}_{i}\right\} \rightarrow \mathbb{R}$ be a function. The partial delta derivative of $f$ respect to $t_{i} \in \mathbb{T}_{i}^{k}$ is defined as the limit

$$
\lim _{\substack{s_{i} \rightarrow t_{i} \\ s_{i} \neq \sigma_{i}\left(t_{i}\right)}} \frac{f\left(t_{1}, t_{2}, \ldots, t_{i-1}, \sigma_{i}\left(t_{i}\right), t_{i+1}, \ldots, t_{n}\right)-f\left(t_{1}, t_{2}, \ldots, t_{i-1}, s_{i}, t_{i+1}, \ldots, t_{n}\right)}{\sigma_{i}\left(t_{i}\right)-s_{i}}=\frac{\partial f(t)}{\Delta_{i} t_{i}} .
$$

Let the function $f: \mathbb{T}_{1} \times \mathbb{T}_{2} \rightarrow \mathbb{R}$ be $\sigma_{1}$-completely delta differentiable at the point $\left(t^{0}, s^{0}\right)$. If the functions $\varphi$ and $\psi$ have delta derivatives at the point $\xi^{0}$, then the composite function

$$
F(\xi)=f(\varphi(\xi), \psi(\xi)) \text { for } \xi \in \mathbb{T}
$$

has a delta derivative at that point which is expressed by the formula

$$
F^{\Delta}\left(\xi^{0}\right)=\frac{\partial f\left(t^{0}, s^{0}\right)}{\Delta_{1} t} \varphi^{\Delta}\left(\xi^{0}\right)+\frac{\partial f\left(\sigma_{1}\left(t^{0}\right), s^{0}\right)}{\Delta_{2} s} \psi^{\Delta}\left(\xi^{0}\right)
$$

where $\left(t^{0}, s^{0}\right)=\left(\varphi\left(\xi^{0}\right), \psi\left(\xi^{0}\right)\right), \sigma_{1}\left(\varphi\left(\xi^{0}\right)\right)=\varphi\left(\sigma\left(\xi^{0}\right)\right)$.

Remark 1. If $\mathbb{T}=\mathbb{R}$ and $F(\xi)=f(\xi, \psi(\xi))$, then (8) means that

$$
F^{\Delta}\left(\xi^{0}\right)=\frac{\partial f\left(\xi^{0}, s^{0}\right)}{\Delta_{1} t}+\frac{\partial f\left(\xi^{0}, s^{0}\right)}{\partial s} \psi^{\Delta}\left(\xi^{0}\right)
$$

Remark 2 . If the function $f: \mathbb{T}_{1} \times \mathbb{T}_{2} \rightarrow \mathbb{R}$ be $\sigma_{2}$-completely delta differentiable at the point $\left(t^{0}, s^{0}\right)$, if the functions $\psi$ and $\varphi$ have delta derivatives at the point $\xi^{0}$, then the composite function

$$
F(\xi)=f(\psi(\xi), \varphi(\xi)) \text { for } \xi \in \mathbb{T}
$$

has a delta derivative at that point which is expressed by the formula

$$
F^{\Delta}\left(\xi^{0}\right)=\frac{\partial f\left(s^{0}, t^{0}\right)}{\Delta_{1} s} \psi^{\Delta}\left(\xi^{0}\right)+\frac{\partial f\left(\sigma_{2}\left(s^{0}\right), t^{0}\right)}{\Delta_{2} t} \varphi^{\Delta}\left(\xi^{0}\right)
$$

where $\left(s^{0}, t^{0}\right)=\left(\psi\left(\xi^{0}\right), \varphi\left(\xi^{0}\right)\right), \sigma_{2}\left(\psi\left(\xi^{0}\right)\right)=\psi\left(\sigma\left(\xi^{0}\right)\right)$.

The formulae (8) and (11) constitute a dual pair in which the shift appears at two different places. This is a natural phenomenon in the calculus on time scales such as the product rule (3) has two forms.

Let a function $f: \mathbb{T}_{1} \times \mathbb{T}_{2} \rightarrow \mathbb{R}$ have the mixed delta derivatives $\partial^{2} f(t, s) / \Delta_{1} t \Delta_{2} s$ and $\partial^{2} f(t, s) / \Delta_{2} s \Delta_{1} t$ in some 
neighborhood of the point $\left(t^{0}, s^{0}\right) \in \mathbb{T}_{1}^{k} \times \mathbb{T}_{2}^{k}$. If these derivatives are continuous at the point $\left(t^{0}, s^{0}\right)$, then

$$
\frac{\partial^{2} f(t, s)}{\Delta_{1} t \Delta_{2} s}=\frac{\partial^{2} f(t, s)}{\Delta_{2} s \Delta_{1} t}
$$

\section{Hamilton Canonical Equations on Time Scales}

In this section, we present the Hamilton canonical equations on time scales that have been discussed in literatures [36-38].

Assuming that the configuration of a mechanical system is determined by generalized coordinate $q$, the Lagrangian $L$ is $L\left(t, q^{\sigma}(t), q^{\Delta}(t)\right)$, where $t \in \mathbb{T}, q:[a, b]_{\mathbf{T}} \rightarrow \mathbb{R}, q \in \mathrm{C}_{\mathrm{rd}}^{1}$. The Hamilton action is

$$
S[q(\cdot)]=\int_{a}^{b} L\left(t, q^{\sigma}, q^{\Delta}\right) \Delta t .
$$

The Hamilton principle on time scales can be expressed as

$$
\delta S=0,
$$

which satisfies the relationships

$$
\begin{aligned}
& \delta q^{\Delta}=(\delta q)^{\Delta}, \\
& \delta q^{\sigma}=(\delta q)^{\sigma},
\end{aligned}
$$

and the boundary conditions

$$
\left.\delta q\right|_{t=a}=\left.\delta q\right|_{t=b}=0 .
$$

The generalized momentum and the Hamiltonian are

$$
\begin{aligned}
p & =\frac{\partial L}{\partial q^{\Delta}}, \\
H & =H\left(p, q^{\sigma}, t\right)=p q^{\Delta}-L .
\end{aligned}
$$

The Hamilton canonical equations on time scales can be derived from the principle above, that is,

$$
\begin{aligned}
q^{\Delta} & =\frac{\partial H}{\partial p}, \\
p^{\Delta} & =-\frac{\partial H}{\partial q^{\sigma}} .
\end{aligned}
$$

\section{Canonical Transformations on Time Scales}

Now, we consider the transformation from the old variables $q, p$ to the new variables $Q, P$

$$
\begin{aligned}
& Q=Q(p, q, t), \\
& P=P(p, q, t),
\end{aligned}
$$

and suppose that (19) is invertible. Undergoing the transformation, the canonical equations (18) on time scales will be transformed into other new equations. However, the new equations may not have the canonical form in general. Among the possible transformations, there is a class of transformations called canonical transformations whose properties are particularly useful in dynamics. That is, the canonical equations (18) are transformed into the new canonical equations

$$
\begin{aligned}
Q^{\Delta} & =\frac{\partial H^{*}}{\partial P}, \\
P^{\Delta} & =-\frac{\partial H^{*}}{\partial Q^{\sigma}},
\end{aligned}
$$

where the new Hamiltonian $H^{*}\left(P, Q^{\sigma}, t\right)$ is

$$
H^{*}\left(P, Q^{\sigma}, t\right)=P Q^{\Delta}-L .
$$

The following criterion gives the condition under which (19) is canonical on time scales.

Criterion 1. If (19) satisfies the condition

$$
p q^{\Delta}-P Q^{\Delta}+H^{*}-H=\frac{\Delta F}{\Delta t},
$$

then it is a canonical transformation on time scales, where $F$ is delta differentiable.

Proof 1. Formula (22) can be written as

$$
p \Delta q-P \Delta Q+\left(H^{*}-H\right) \Delta t=\Delta F,
$$

suppose that $q$ and $p$ exist with the isochronal variations $\delta q$ and $\delta p$. Then (23) becomes

$$
p \delta q-P \delta Q=\delta F .
$$

Taking the delta derivative of (24) with respect to $t$, we obtain

$$
p^{\Delta} \delta q+p^{\sigma}(\delta q)^{\Delta}-P^{\Delta} \delta Q-P^{\sigma}(\delta Q)^{\Delta}=(\delta F)^{\Delta} .
$$

Taking the variation of (22), we have

$$
q^{\Delta} \delta p+p \delta q^{\Delta}-Q^{\Delta} \delta P-P \delta Q^{\Delta}+\delta H^{*}-\delta H=\delta F^{\Delta} .
$$

By using (15) and subtracting (25) from (26) yields

$$
\begin{aligned}
& \left(q^{\Delta} \delta p-p^{\Delta} \delta q\right)-\left(Q^{\Delta} \delta P-P^{\Delta} \delta Q\right)+\delta H^{*} \\
& \quad-\delta H+\left(p-p^{\sigma}\right)(\delta q)^{\Delta}-\left(P-P^{\sigma}\right)(\delta Q)^{\Delta}=0 .
\end{aligned}
$$

According to (3), (27) becomes

$$
\left(q^{\Delta} \delta p-p^{\Delta} \delta q^{\sigma}\right)-\left(Q^{\Delta} \delta P-P^{\Delta} \delta Q^{\sigma}\right)+\delta H^{*}-\delta H=0 .
$$

Taking note of (18), we obtain

$$
\left(Q^{\Delta} \delta P-P^{\Delta} \delta Q^{\sigma}\right)-\delta H^{*}=0 .
$$

Therefore, (20) can be derived from (29).

In fact, (22) is also the necessary condition of the canonical transformation on time scales. According to the Hamilton principle on time scales, if both the new variables and old variables satisfy 


$$
\begin{gathered}
\delta \int_{a}^{b}\left[P Q^{\Delta}-H^{*}\left(P, Q^{\sigma}, t\right)\right] \Delta t=0, \\
\delta \int_{a}^{b}\left[p q^{\Delta}-H\left(p, q^{\sigma}, t\right)\right] \Delta t=0,
\end{gathered}
$$

then (19) is canonical on time scales. From (30) and (31), the two integrands should satisfy the following relation

$$
\left[p q^{\Delta}-H\left(p, q^{\sigma}, t\right)\right]-\left[P Q^{\Delta}-H^{*}\left(P, Q^{\sigma}, t\right)\right]=\frac{\Delta F}{\Delta t},
$$

where $F$ can be an arbitrary function with new variables, old variables, and time.

Because the canonical transformation completely depends on the choice of the arbitrary function $F$, the function $F$ can be called the generating function. Clearly, $F$ and $H^{*}$ can be solved from (23), and the solution of the canonical transformation problem on time scales is not unique. In order to achieve the transformation between the two groups of canonical variables, the generating function $F$ should contain both new variables and old variables [1]. With that case in mind, the four forms of the canonical transformations on time scales are discussed below, and some simple and important examples are given to illustrate the effect of the canonical transformation on time scales and the generating function.

Case 1. The generating function of the first kind has the form

$$
F=F_{1}(Q, q, t)
$$

we have

$$
\begin{aligned}
\Delta F= & \frac{\partial F_{1}(Q, q, t)}{\Delta_{1} Q} \Delta Q+\frac{\partial F_{1}\left(Q^{\sigma}, q, t\right)}{\Delta_{2} q} \Delta q \\
+ & \frac{\partial F_{1}\left(Q^{\sigma}, q^{\sigma}, t\right)}{\Delta_{3} t} \Delta t .
\end{aligned}
$$

Substituting (34) into (23), we obtain

$$
\begin{gathered}
-\left(P+\frac{\partial F_{1}(Q, q, t)}{\Delta_{1} Q}\right) \Delta Q+\left(p-\frac{\partial F_{1}\left(Q^{\sigma}, q, t\right)}{\Delta_{2} q}\right) \Delta q \\
+\left(H^{*}-H-\frac{\partial F_{1}\left(Q^{\sigma}, q^{\sigma}, t\right)}{\Delta_{3} t}\right) \Delta t=0 .
\end{gathered}
$$

From (35), we can find

$$
\begin{aligned}
P & =-\frac{\partial F_{1}(Q, q, t)}{\Delta_{1} Q}, \\
p & =\frac{\partial F_{1}\left(Q^{\sigma}, q, t\right)}{\Delta_{2} q}, \\
H^{*} & =H+\frac{\partial F_{1}\left(Q^{\sigma}, q^{\sigma}, t\right)}{\Delta_{3} t} .
\end{aligned}
$$

Example 1. If the generating function has the form

$$
F_{1}=q Q
$$

then the corresponding canonical transformation gives

$$
\begin{aligned}
p & =Q^{\sigma}, \\
P & =-q, \\
H^{*} & =H .
\end{aligned}
$$

The transformation (38) shows that the new generalized coordinates depend on the old generalized momentum while the new generalized momentum depends on the old generalized coordinates.

Case 2. The generating function of the second kind has the form

$$
F=F_{2}(P, q, t)-Q P
$$

combining (39) and (23), we have

$$
\begin{aligned}
\left(Q^{\sigma}-\frac{\partial F_{2}(P, q, t)}{\Delta_{1} P}\right) \Delta P & +\left(p-\frac{\partial F_{2}\left(P^{\sigma}, q, t\right)}{\Delta_{2} q}\right) \Delta q \\
& +\left(H^{*}-H-\frac{\partial F_{2}\left(P^{\sigma}, q^{\sigma}, t\right)}{\Delta_{3} t}\right) \Delta t=0 .
\end{aligned}
$$

From (40), we obtain

$$
\begin{aligned}
Q^{\sigma} & =\frac{\partial F_{2}(P, q, t)}{\Delta_{1} P}, \\
p & =\frac{\partial F_{2}\left(P^{\sigma}, q, t\right)}{\Delta_{2} q}, \\
H^{*} & =H+\frac{\partial F_{2}\left(P^{\sigma}, q^{\sigma}, t\right)}{\Delta_{3} t} .
\end{aligned}
$$

Example 2. If the generating function has the form

$$
F_{2}=P q,
$$

then the corresponding canonical transformation gives

$$
\begin{aligned}
p & =P^{\sigma}, \\
Q^{\sigma} & =q, \\
H^{*} & =H .
\end{aligned}
$$

Case 3. The generating function of the third kind has the form

$$
F=F_{3}(Q, p, t)+p q
$$

Similarly, we have

$$
\begin{gathered}
P=-\frac{\partial F_{3}(Q, p, t)}{\Delta_{1} Q}, \\
q^{\sigma}=-\frac{\partial F_{3}\left(Q^{\sigma}, p, t\right)}{\Delta_{2} p}, \\
H^{*}=H+\frac{\partial F_{3}(Q, p, t)}{\Delta_{3} t} .
\end{gathered}
$$

Example 3. If the generating function has the form

$$
F_{3}=-p Q
$$

then the corresponding canonical transformation gives 


$$
\begin{aligned}
q^{\sigma} & =Q^{\sigma}, \\
P & =p, \\
H^{*} & =H .
\end{aligned}
$$

The transformation (47) shows that the new generalized coordinates and the new generalized momentum are the same with the old ones. Hence, the generating function (46) is corresponding to the identical transformation.

Case 4. The generating function of the fourth kind has the form

$$
F=F_{4}(P, p, t)+p q-P Q .
$$

Similarly, we have

$$
\begin{aligned}
Q^{\sigma} & =\frac{\partial F_{4}(P, p, t)}{\Delta_{1} P}, \\
q^{\sigma} & =-\frac{\partial F_{4}\left(P^{\sigma}, p, t\right)}{\Delta_{2} p}, \\
H^{*} & =H+\frac{\partial F_{4}\left(P^{\sigma}, p^{\sigma}, t\right)}{\Delta_{3} t} .
\end{aligned}
$$

Example 4. If the generating function has the form

$$
F_{4}=p P,
$$

then the corresponding canonical transformation gives

$$
\begin{aligned}
q^{\sigma} & =-P^{\sigma}, \\
Q^{\sigma} & =p, \\
H^{*} & =H .
\end{aligned}
$$

Compared with (38), (51) is actually the same with (38). Hence, different generating functions can correspond to the same canonical transformation.

The canonical transformations expressed by the four kinds of generating functions above are only part of the canonical transformations on time scales. However, it is quite extensive. If $t$ is not explicitly contained in $F_{1}, F_{2}, F_{3}$, and $F_{4}$, then $H^{*}=H$, (23) can be written as

$$
p \Delta q-P \Delta Q=\Delta F
$$

This kind of transformation can be called contact transformation on time scales.

\section{Hamilton-Jacobi Method on Time Scales}

The form of Hamiltonian will be simpler if we choose an appropriate generating function, such as $H^{*}=0$. If $H^{*}=0$, (20) becomes

$$
\begin{aligned}
& Q^{\Delta}=\frac{\partial H^{*}}{\partial P}=0, \\
& P^{\Delta}=-\frac{\partial H^{*}}{\partial Q^{\sigma}}=0 .
\end{aligned}
$$

By integrating, we have

$$
\begin{aligned}
& Q=\alpha, \\
& P=\beta,
\end{aligned}
$$

where $\alpha$ and $\beta$ are constants and $\alpha=\alpha^{\sigma}, \beta=\beta^{\sigma}$.

In order to achieve the purpose above depends on the generating function we choose. According to the relationship between the new Hamiltonian and the old Hamiltonian

$$
H^{*}=H+\frac{\partial F}{\Delta t} .
$$

Thus, the generating function should satisfy

$$
H+\frac{\partial F}{\Delta t}=0 .
$$

If we choose $F=F_{2}(P, q, t)-Q P$ and use symbol $S$ take the place of $F_{2}$, then we can obtain the following equation

$$
H\left(p, q^{\sigma}, t\right)+\frac{\partial S\left(P^{\sigma}, q^{\sigma}, t\right)}{\Delta_{3} t}=0 .
$$

Substituting $P=\beta$ into $S$, we have

$$
S=S(\beta, q, t) .
$$

Obviously, $\beta$ can be determined by initial conditions. Hence, the transformation relation corresponding to the function $S=F_{2}(P, q, t)$ can be written as

$$
\begin{aligned}
& \alpha=\frac{\partial S(\beta, q, t)}{\Delta_{1} \beta}, \\
& p=\frac{\partial S(\beta, q, t)}{\Delta_{2} q} .
\end{aligned}
$$

Substituting $p$ into (57), we obtain

$$
H\left(\frac{\partial S(\beta, q, t)}{\Delta_{2} q}, q^{\sigma}, t\right)+\frac{\partial S\left(\beta, q^{\sigma}, t\right)}{\Delta_{3} t}=0 .
$$

Equation (60) can be called the Hamilton-Jacobi equation on time scales.

Theorem 1. If function $S(\beta, q, t)$ is a complete integral of the Hamilton-Jacobi equation (60) on time scales, that is, $S \in \mathrm{C}_{\mathrm{rd}}^{2}, \beta$ is the constant of integration, and

$$
\operatorname{det}\left(\frac{\partial^{2} S(\beta, q, t)}{\Delta_{1} \beta \Delta_{2} q}\right) \neq 0,
$$

and it satisfies the Hamilton-Jacobi equation (60) on time scales, then the first integrals of the canonical equations (18) on time scales are determined by 


$$
\begin{gathered}
\alpha=\frac{\partial S(\beta, q, t)}{\Delta_{1} \beta}, \\
p=\frac{\partial S(\beta, q, t)}{\Delta_{2} q} .
\end{gathered}
$$

Proof 2. First, we show that (62) is the first integral of the system on time scales, namely,

$$
\frac{\partial^{2} S(\beta, q, t)}{\Delta_{1} \beta \Delta_{2} q} q^{\Delta}+\frac{\partial^{2} S\left(\beta, q^{\sigma}, t\right)}{\Delta_{1} \beta \Delta_{3} t}=0 .
$$

Taking note of (18) and (64), we have

$$
\frac{\partial^{2} S(\beta, q, t)}{\Delta_{1} \beta \Delta_{2} q} \frac{\partial H\left(\left(S(\beta, q, t) / \Delta_{2} q\right), q^{\sigma}, t\right)}{\partial\left(S(\beta, q, t) / \Delta_{2} q\right)}+\frac{\partial^{2} S\left(\beta, q^{\sigma}, t\right)}{\Delta_{1} \beta \Delta_{3} t}=0 .
$$

Taking the partial delta derivative of (60) with respect to $\beta$ and noting (9), we have

$$
\frac{\partial H\left(\left(\partial S(\beta, q, t) / \Delta_{2} q\right), q^{\sigma}, t\right)}{\partial\left(\partial S(\beta, q, t) / \Delta_{2} q\right)} \frac{\partial^{2} S(\beta, q, t)}{\Delta_{2} q \Delta_{1} \beta}+\frac{\partial^{2} S\left(\beta, q^{\sigma}, t\right)}{\Delta_{3} t \Delta_{1} \beta}=0
$$

Notice that (66) coincides with (65). Therefore, (64) is an identity.

Similarly, we verify that (63) is also the first integral. Actually, taking the delta derivative of (63) with respect to $t$, we have

$$
p^{\Delta}=\frac{\partial^{2} S(\beta, q, t)}{\Delta_{2} q \Delta_{2} q} q^{\Delta}+\frac{\partial^{2} S\left(\beta, q^{\sigma}, t\right)}{\Delta_{2} q^{\sigma} \Delta_{3} t} .
$$

Then (67) becomes

$$
\begin{aligned}
-p^{\Delta}+ & \frac{\partial^{2} S(\beta, q, t)}{\Delta_{2} q \Delta_{2} q} \frac{\partial H\left(\left(S(\beta, q, t) / \Delta_{2} q\right), q^{\sigma}, t\right)}{\partial\left(S(\beta, q, t) / \Delta_{2} q\right)} \\
& +\frac{\partial^{2} S\left(\beta, q^{\sigma}, t\right)}{\Delta_{2} q^{\sigma} \Delta_{3} t}=0 .
\end{aligned}
$$

Taking the partial delta derivative of (60) with respect to $q$ yields

$$
\begin{gathered}
\frac{\partial H\left(\left(\partial S(\beta, q, t) / \Delta_{2} q\right), q^{\sigma}, t\right)}{\partial\left(\partial S(\beta, q, t) / \Delta_{2} q\right)} \frac{\partial^{2} S(\beta, q, t)}{\Delta_{2} q \Delta_{2} q} \\
+\frac{\partial H}{\partial q^{\sigma}}+\frac{\partial^{2} S\left(\beta, q^{\sigma}, t\right)}{\Delta_{3} t \Delta_{2} q^{\sigma}}=0 .
\end{gathered}
$$

Considering $S \in \mathrm{C}_{\mathrm{rd}}^{2}$ and (18), we know that (69) coincides with (68), that is, (68) is also an identity, completing the proof.

Example 5. The Emden-Fowler type equations have significant applications in gas dynamics, fluid mechanics, relativistic mechanics, and nuclear physics and also play an important role in the study of symmetries and dynamical inverse problems.
Different kinds of Emden-Fowler type equations in version of time scales have been established and many achievements have been made [43-45].

Now, we investigate the integrals of an Emden-Fowler type equation on time scales. Assume that the Hamiltonian of the system on time scales has the following form

$$
H=\frac{1}{2}\left[p^{2} \frac{1}{t \sigma(t)}+\frac{a}{m+2} \frac{t^{m+2}}{\sigma(t)}\left(q^{\sigma}\right)^{2 m+4}\right],
$$

where $a$ and $m$ are constant. The Hamilton canonical equations of system on time scales are

$$
\begin{aligned}
& q^{\Delta}=\frac{\partial H}{\partial p}=\frac{p}{t \sigma(t)} \\
& p^{\Delta}=-\frac{\partial H}{\partial q^{\sigma}}=-a \frac{t^{m+2}}{\sigma(t)}\left(q^{\sigma}\right)^{2 m+3} .
\end{aligned}
$$

When $a=1, m=1$, and $\mathbb{T}=\mathbb{R}$, the equations lead to the classical Emden equation

$$
t \ddot{q}+2 \dot{q}+t q^{5}=0 .
$$

The Hamilton-Jacobi equation on time scales is given by

$$
\begin{aligned}
& \frac{\partial S\left(\beta, q^{\sigma}, t\right)}{\Delta_{3} t}+\frac{1}{2} \\
& \quad\left[\frac{1}{t(t+h)}\left(\frac{\partial S(\beta, q, t)}{\Delta_{2} q}\right)^{2}+\frac{a}{m+2} \frac{t^{m+2}}{t+h}\left(q^{\sigma}\right)^{2 m+4}\right]=0 .
\end{aligned}
$$

Equation (73) is hard to solve by the method of variable separation. Now, assume $m=-3$ and $t \in \mathbb{T}=h \mathbb{Z}, h>$ 0 , then $\sigma(t)=t+h, \mu(t)=h$. Thus, (73) becomes

$$
\frac{\partial S\left(\beta, q^{\sigma}, t\right)}{\Delta_{3} t}+\frac{1}{2 t(t+h)}\left[\left(\frac{\partial S(\beta, q, t)}{\Delta_{2} q}\right)^{2}-a\left(q^{\sigma}\right)^{-2}\right]=0 .
$$

By using the method of variable separation, we set the complete integral as

$$
S=S_{1}(t)+S_{2}(q)
$$

Substituting (75) into (74), we have

$$
2 t(t+h) \frac{\partial S_{1}(t)}{\Delta_{3} t}=a\left(q^{\sigma}\right)^{-2}-\left(\frac{\partial S_{2}(q)}{\Delta_{2} q}\right)^{2}=\beta
$$

By integrating (76), we obtain

$$
\begin{aligned}
S_{1} & =-\frac{\beta}{2 t}, \\
S_{2} & =\int\left(a\left(q+h q^{\Delta}\right)^{-2}-\beta\right)^{1 / 2} \Delta_{2} q, \\
S & =-\frac{\beta}{2 t}+\int\left(a\left(q+h q^{\Delta}\right)^{-2}-\beta\right)^{1 / 2} \Delta_{2} q,
\end{aligned}
$$

and 


$$
\begin{aligned}
& \alpha=\frac{\partial S(\beta, q, t)}{\Delta_{1} \beta}=-\frac{1}{2 t}-\frac{1}{2} \int\left(a\left(q+h q^{\Delta}\right)^{-2}-\beta\right)^{-(1 / 2)} \Delta_{2} q, \\
& p=\frac{\partial S(\beta, q, t)}{\Delta_{2} q}=\frac{\partial S_{2}(q)}{\Delta_{2} q}=\int \frac{\left(a\left(q(t+h)+h q^{\Delta}(t+h)\right)^{-2}-\beta\right)^{1 / 2}-\left(a\left(q(t)+h q^{\Delta}(t)\right)^{-2}-\beta\right)^{1 / 2}}{q(t+h)-q(t)} \Delta_{2} q .
\end{aligned}
$$

In this example, we gave an Emden-Fowler type of equation (71) on time scales which was a specific case of the general equation in [44]. By applying Theorem 1, we found the integrals of (71) when $\mathbb{T}=h \mathbb{Z}$.

The Emden model on time scales contains not only continuous and discrete cases but also other more general cases. More potential applications for the Emden model on time scales in symmetries, dynamical inverse problems and oscillations, and control are worth looking forward to.

\section{Conclusions}

In this paper, we presented the Hamilton-Jacobi method for mechanical systems on time scales. We started from the canonical transformation theory on time scales. Four examples were given to show the role played by a generating function in the canonical transformation. Then we constructed the Hamilton-Jacobi equation (60) on time scales and proved the Jacobi theorem on time scales. We illustrated how this method works for dynamical equations on time scales. The results of this paper are more general. The continuous and discrete analogues of the Hamilton-Jacobi method are special cases of this paper. Moreover, this method can also be applied to the constrained mechanical systems on time scales, the symplectic dynamical systems on time scales, and the impulsive dynamical systems on time scales.

From a geometrical point of view, the Hamilton-Jacobi theory on time scales remains an interesting open question. Further works about finding the integral of dynamical equations on time scales are still worth doing, for example, the Poisson theory on time scales and the Lie symmetry theory on time scales.

\section{Data Availability}

The numerical data analysed during the present study used to support the findings of this study are available from the corresponding author upon reasonable request.

\section{Conflicts of Interest}

There are no conflicts of interest regarding this research work.

\section{Authors' Contributions}

All authors contributed equally to this manuscript and have read and approved the final version.

\section{Acknowledgments}

This work was supported by the National Natural Science Foundation of China (Grant nos. 11572212, 11272227).

\section{References}

[1] H. Goldstein, C. Poole, and J. Safko, Classical Mechanics, Higher Education Press, Beijing, China, 2005.

[2] V. I. Arnold, Mathematical Methods of Classical Mechanics, Springer, New York, NY, USA, 1991.

[3] B. D. Vujanović, "Application of the Hamilton-Jacobi method to the study of rheo-linear oscillators," Acta Mechanica, vol. 93, no. 1-4, pp. 179-190, 1992.

[4] C. Lin and M. M. Lin, "The research of the quantum Hamilton-Jacobi theory," Communications in Nonlinear Science and Numerical Simulation, vol. 14, no. 5, pp. 1958-1961, 2009.

[5] S. E. Dreyfus, "Dynamic programming and the HamiltonJacobi method of classical mechanics," Journal of Optimization Theory and Applications, vol. 2, no. 1, pp. 15-26, 1968.

[6] M. S. Marinov, "An alternative to the Hamilton-Jacobi approach in classical mechanics," Journal of Physics A: Mathematical and General, vol. 12, no. 1, pp. 31-47, 1979.

[7] N. D. An, "One method for solving the Hamilton-Jacobi equations in analytical mechanics," Ukrainian Mathematical Journal, vol. 36, no. 1, pp. 110-112, 1984.

[8] B. D. Vujanović and T. M. Atanacković, An Introduction to Modern Variational Techniques in Mechanics and Engineering, Birkhäuser, Boston, MA, USA, 2004.

[9] M. de León, J. C. Marrero, and D. M. de Diego, "Linear almost Poisson structures and Hamilton-Jacobi equation. Applications to nonholonomic mechanics," Journal of Geometric Mechanics, vol. 2, no. 2, pp. 159-198, 2010.

[10] T. Ohsawa and A. M. Bloch, "Nonholonomic Hamilton-Jacobi equation and integrability," Journal of Geometric Mechanics, vol. 1, no. 4, pp. 461-481, 2009.

[11] M. Leok, T. Ohsawa, and D. Sosa, "Hamilton-Jacobi theory for degenerate Lagrangian systems with holonomic and nonholonomic constraints," Journal of Mathematical Physics, vol. 53, no. 7, article 072905, 2012.

[12] Y. Wang, F. X. Mei, J. Xiao, and Y. X. Guo, "A kind of nonconservative Hamilton system solved by the Hamilton-Jacobi method," Acta Physica Sinica, vol. 66, article 054501, 2017.

[13] T. Ohsawa, O. E. Fernandez, A. M. Bloch, and D. V. Zenkov, "Nonholonomic Hamilton-Jacobi theory via Chaplygin Hamiltonization," Journal of Geometry and Physics, vol. 61, no. 8, pp. 1263-1291, 2011.

[14] F. X. Mei, H. B. Wu, and Y. F. Zhang, "Hamilton-Jacobi method for solving ordinary differential equations," Chinese Physics, vol. 15, no. 8, pp. 1662-1664, 2006. 
[15] S. A. Hojman, "Construction of Lagrangian and Hamiltonian structures starting from one constant of motion," Acta Mechanica, vol. 226, no. 3, pp. 735-744, 2015.

[16] N. Elnatanov and J. Schiff, "The Hamilton-Jacobi difference equation," Functional Differential Equations, vol. 3, pp. 279286, 1996.

[17] S. Lall and M. West, "Discrete variational Hamiltonian mechanics," Journal of Physics A: Mathematical and General, vol. 39, no. 19, pp. 5509-5519, 2006.

[18] T. Ohsawa, A. M. Bloch, and M. Leok, "Discrete HamiltonJacobi theory and discrete optimal control," in 49th IEEE Conference on Decision and Control (CDC), pp. 5438-5443, Atlanta, GA, USA, 2010.

[19] S. Hilger, Ein Maßkettenkalkül mit Anwendung auf Zentrumsmannigfaltigkeiten, [Ph.D. thesis], Universtät Würzburg, 1988.

[20] W. G. Kelly and A. Peterson, Difference Equations: An Introduction with Applications, Academic Press, New York, NY, USA, 1991.

[21] M. Bohner and A. Peterson, Dynamic Equations on Time Scale: An Introduction with Applications, Birkhäuser, Boston, MA, USA, 2001.

[22] R. Agarwal, M. Bohner, D. O'Regan, and A. Peterson, "Dynamic equations on time scales: a survey," Journal of Computational and Applied Mathematics, vol. 141, no. 1-2, pp. 1-26, 2002.

[23] F. M. Atici, D. C. Biles, and A. Lebedinsky, "An application of time scales to economics," Mathematical and Computer Modelling, vol. 43, no. 7-8, pp. 718-726, 2006.

[24] M. Bohner and S. G. Georgiev, Multivariable Dynamic Calculus on Time Scales, Springer, Cham, Switzerland, 2016.

[25] M. Bohner, "Calculus of variations on time scales," Dynamic Systems and Applications, vol. 13, no. 12, pp. 339-349, 2004.

[26] R. Hilscher and V. Zeidan, "Calculus of variations on time scales: weak local piecewise $C_{\text {rd }}{ }^{1}$ solutions with variable endpoints," Journal of Mathematical Analysis and Applications, vol. 289, no. 1, pp. 143-166, 2004.

[27] M. Bohner and G. S. Guseinov, "Double integral calculus of variations on time scales," Computers \& Mathematics with Applications, vol. 54, no. 1, pp. 45-57, 2007.

[28] N. Martins and D. F. M. Torres, "Calculus of variations on time scales with nabla derivatives," Nonlinear Analysis: Theory, Methods \& Applications, vol. 71, no. 12, pp. e763-e773, 2009.

[29] J. Cresson, A. B. Malinowska, and D. F. M. Torres, "Time scale differential, integral, and variational embeddings of Lagrangian systems," Computers \& Mathematics with Applications, vol. 64, no. 7, pp. 2294-2301, 2012.

[30] R. Š. Hilscher and V. Zeidan, "First order conditions for generalized variational problems over time scales," Computers \& Mathematics with Applications, vol. 62, no. 9, pp. 3490-3503, 2011.

[31] R. Almeida and D. F. M. Torres, "Isoperimetric problems on time scales with nabla derivatives," Journal of Vibration and Control, vol. 15, no. 6, pp. 951-958, 2009.

[32] A. B. Malinowska and D. F. M. Torres, "Necessary and sufficient conditions for local Pareto optimality on time scales," Journal of Mathematical Sciences, vol. 161, no. 6, pp. 803810, 2009.

[33] M. Dryl and D. F. M. Torres, "The delta-nabla calculus of variations for composition functionals on time scales,"
International Journal of Difference Equations, vol. 8, no. 1, pp. 27-47, 2013.

[34] Z. Bartosiewicz, N. Martins, and D. F. M. Torres, "The second Euler-Lagrange equation of variational calculus on time scales," European Journal of Control, vol. 17, no. 1, pp. 9-18, 2011.

[35] A. B. Malinowska and D. F. M. Torres, "Euler-Lagrange equations for composition functionals in calculus of variations on time scales," Discrete \& Continuous Dynamical Systems Series $A$, vol. 29, no. 2, pp. 577-593, 2011.

[36] C. D. Ahlbrandt, M. Bohner, and J. Ridenhour, "Hamiltonian systems on time scales," Journal of Mathematical Analysis and Applications, vol. 250, no. 2, pp. 561-578, 2000.

[37] Y. Zhang, "Noether theory for Hamiltonian system on time scales," Chinese Quarterly of Mechanics, vol. 37, no. 2, pp. 214-224, 2016.

[38] C. J. Song and Y. Zhang, "Conserved quantities for Hamiltonian systems on time scales," Applied Mathematics and Computation, vol. 313, pp. 24-36, 2017.

[39] C. J. Song and Y. Zhang, "Noether theorem for Birkhoffian systems on time scales," Journal of Mathematical Physics, vol. 56, no. 10, article 102701, 2015.

[40] Z. Bartosiewicz and D. F. M. Torres, "Noether's theorem on time scales," Journal of Mathematical Analysis and Applications, vol. 342, no. 2, pp. 1220-1226, 2008.

[41] P. P. Cai, J. L. Fu, and Y. X. Guo, "Noether symmetries of the nonconservative and nonholonomic systems on time scales," Science China Physics, Mechanics and Astronomy, vol. 56, no. 5, pp. 1017-1028, 2013.

[42] X. H. Zhai and Y. Zhang, "Noether theorem for nonconservative systems with time delay on time scales," Communications in Nonlinear Science and Numerical Simulation, vol. 52, pp. 32-43, 2017.

[43] M. Bohner, L. Erbe, and A. Peterson, "Oscillation for nonlinear second order dynamic equations on a time scale," Journal of Mathematical Analysis and Applications, vol. 301, no. 2, pp. 491-507, 2005.

[44] S. H. Saker, R. P. Agarwal, and D. O'Regan, "Oscillation of second-order damped dynamic equations on time scales," Journal of Mathematical Analysis and Applications, vol. 330, no. 2, pp. 1317-1337, 2007.

[45] L. Erbe, T. S. Hassan, and A. Peterson, "Oscillation criteria for nonlinear damped dynamic equations on time scales," Applied Mathematics and Computation, vol. 203, no. 1, pp. 343-357, 2008. 


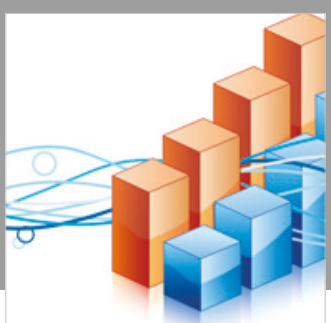

Advances in

Operations Research

\section{-n-m}
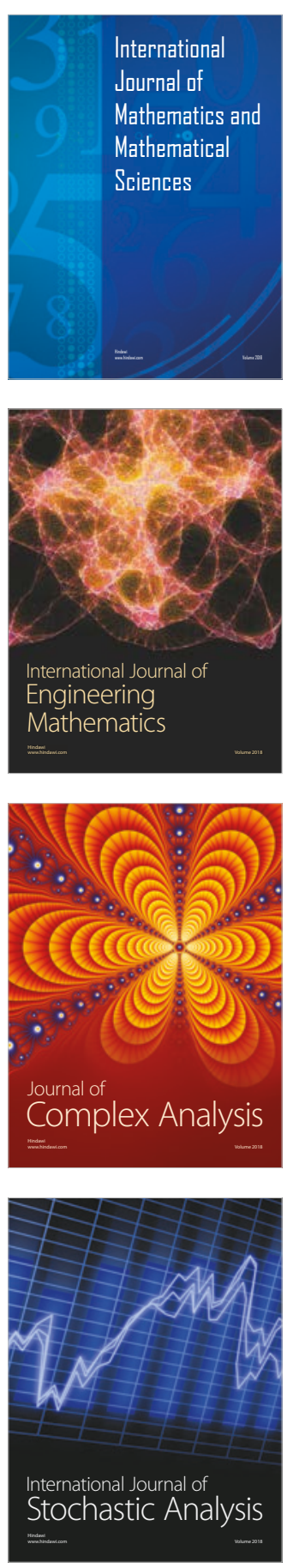
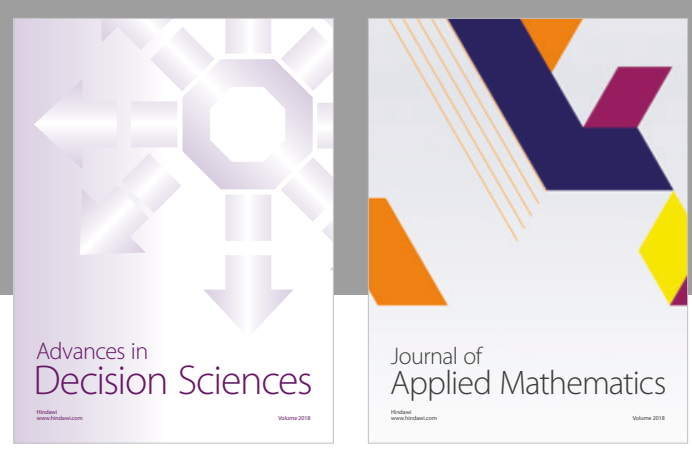

Journal of

Applied Mathematics
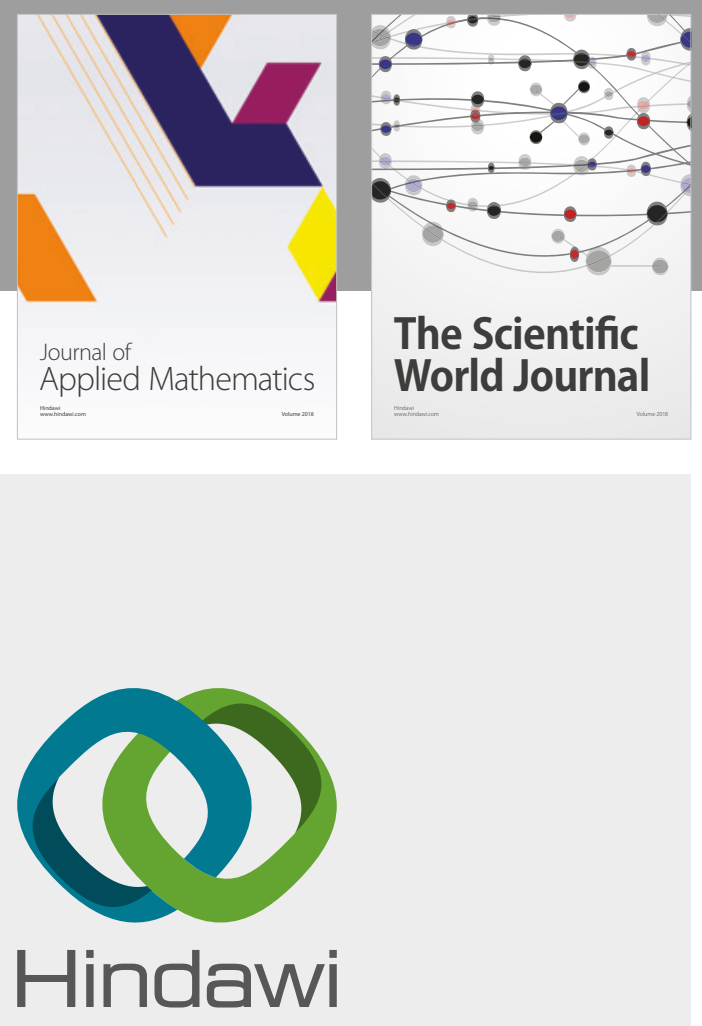

Submit your manuscripts at

www.hindawi.com

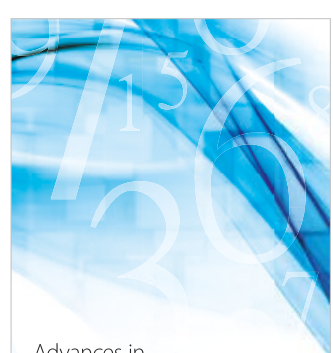

Advances in
Numerical Analysis
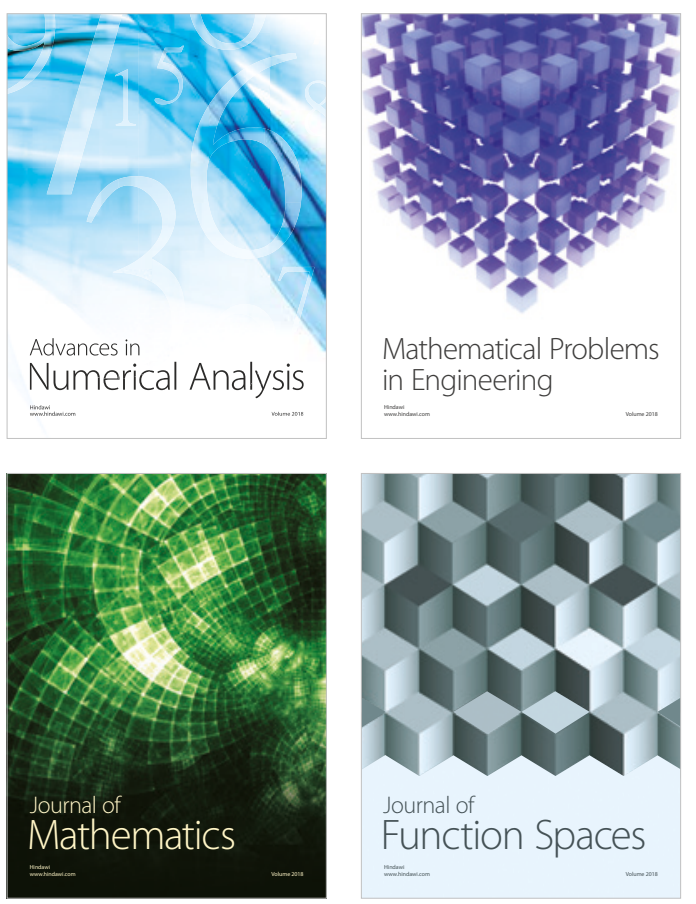

Mathematical Problems in Engineering

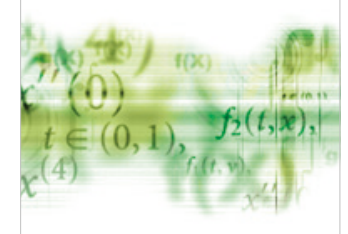

International Journal of

Differential Equations

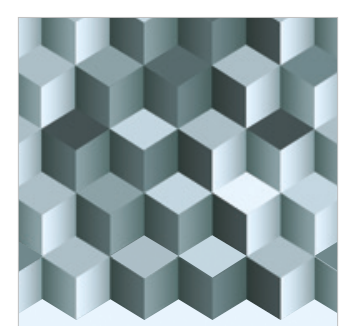

Journal of

Function Spaces
The Scientific

World Journal

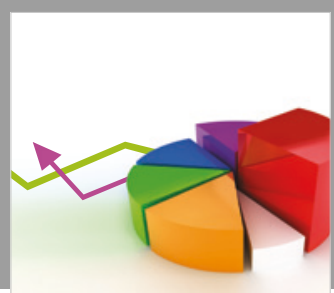

Journal of

Probability and Statistics
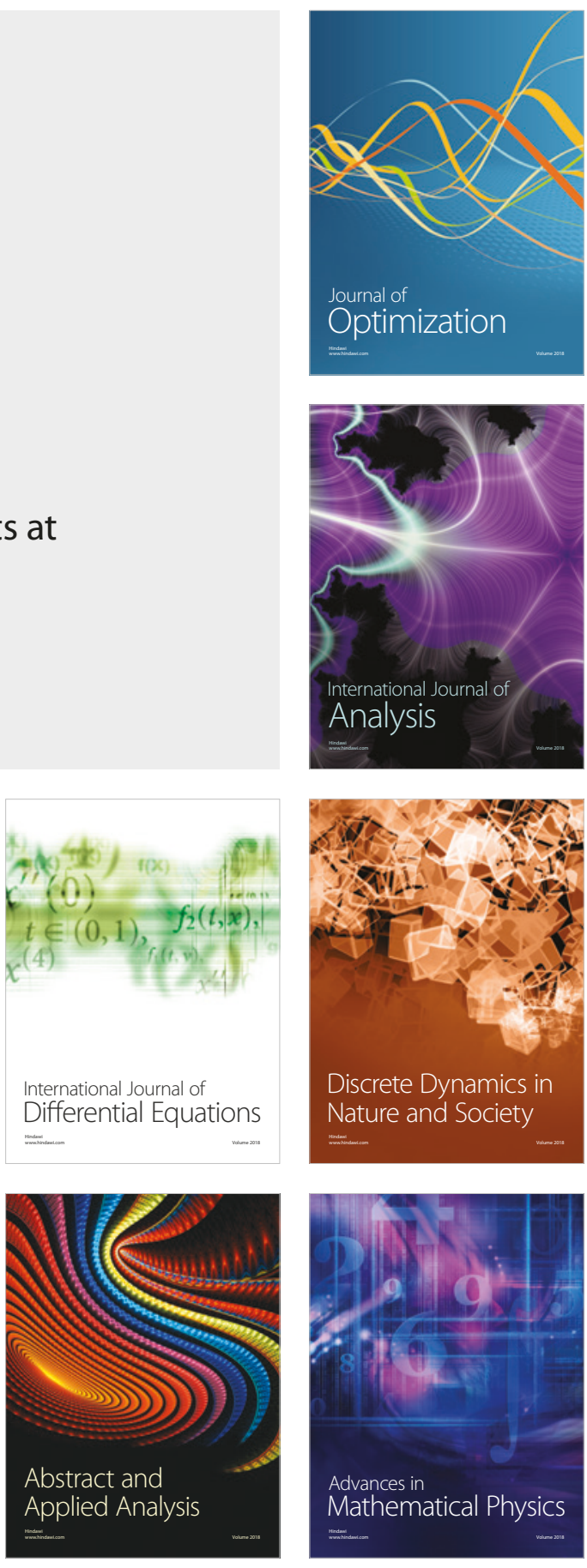